\title{
Identification of genetic variants linked with DCM using cardiac MRI
}

A new genome-wide association study (GWAS) by Krishna Aragam and colleagues that analysed cardiac MRI-derived structural and functional data from UK Biobank participants has identified common variants at 45 genetic loci that contribute to the risk of dilated cardiomyopathy (DCM). "Our results suggest that genetic analyses of imaging-based measures of cardiac structure and function represent a viable strategy that is entirely complementary to growing, case-control GWAS to advance our understanding of heart failure genetics," comments Aragam.

Several case-control GWAS have so far identified genetic variants that are strongly associated with DCM. However, the yield of associated loci in such studies has been limited by modest sample sizes ( $<5,000$ cases). "This research is needed to understand the causes of DCM, whereby conventional Mendelian analysis of genes can only account for $30-40 \%$ of causation," explains Ray Hershberger, who was not involved in the current study. "Given that GWAS need large numbers, and cardiac MRI provides highly accurate measurements of left ventricular size and function, the UK Biobank study is an ideal starting place for this type of genetic analysis," he adds.

The cardiac MRI data of 36,041 UK Biobank participants were assessed in a series of GWAS to identify genetic variants associated with cardiac MRI-derived phenotypes. In total, 57 genetic loci in the human genome were found to associate with cardiac size and function, 45 of which had not been previously described in genetic analyses of DCM. The investigators constructed a polygenic score using the genetic variants that were most robustly associated with left ventricular structure and function. "Our polygenic score influenced the

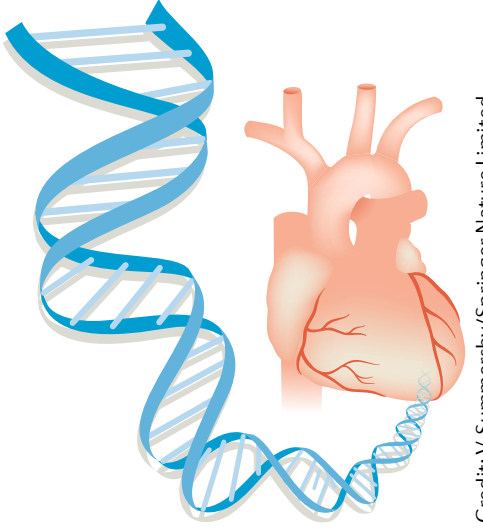

size and function of the left ventricle even among those individuals with rare mutations in the TTN gene, the most frequently identified rare variant mutations among patients with DCM," explains Aragam. "Together, these findings demonstrate that common genetic variants can contribute, in aggregate, to the risk of DCM."

Karina Huynh

with left

ventricular

structure and

function

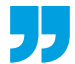

\section{Improved gut microbiota profile in individuals with obesity taking statins}

Individuals with obesity have an increased prevalence of the Bacteroides 2 (Bact2) enterotype, one of four classifications of gut microbiota composition that is associated with systemic inflammation and disease. However, individuals with obesity who are taking statin therapy have a lower prevalence of this dysbiosis than individuals with obesity who are not receiving statins. Causality has not been established, but the possibility

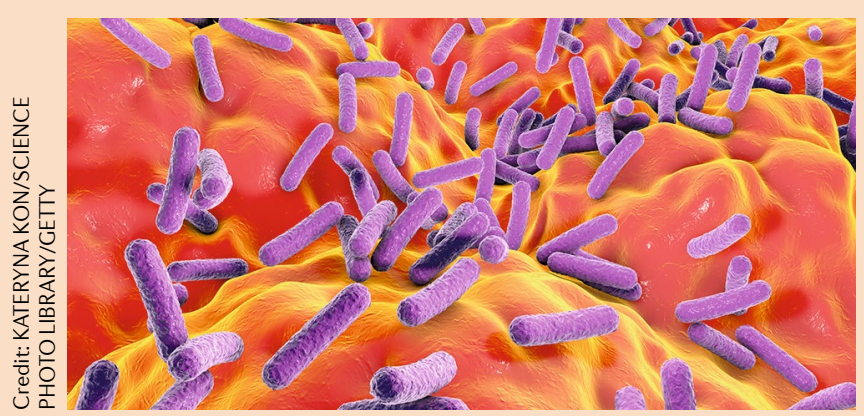

that statin therapy might have gut microbiota-modulatory benefits should now be tested.

Alterations in gut microbiota have been linked with cardiovascular might have gut microbiotamodulatory benefits MetaCardis Body Mass Index Spectrum cohort. Among the individuals who were not taking statins, those with obesity $(\mathrm{BMI} \geq 30)$ had a higher prevalence of the Bact2 enterotype $(17.7 \%)$ than those who were lean or overweight (BMI<30; 3.9\%). The Bact2 enterotype is characterized by a high proportion of Bacteroides and a low proportion of Faecalibacterium. Individuals with this enterotype tend to have loose stools and a higher plasma concentration of the inflammatory marker C-reactive protein than individuals with other enterotypes.
The researchers also found that individuals with obesity who were receiving statin therapy had a lower prevalence of the Bact2 enterotype (5.9\%) than individuals with obesity who were not taking statins (17.7\%). This finding was validated in an independent cohort of 2,345 individuals from the Flemish Gut Flora Project.

These findings raise the intriguing possibility that statin therapy might have a beneficial modulatory effect on the gut microbiota. However, causality cannot be determined from the data reported because, for example, statin users might also have engaged in other health-promoting behaviours. In an accompanying News \& Views article, Peter Libby calls for "a large-scale clinical trial to determine whether statins lead to a reduced prevalence of the Bact2 enterotype in obese participants who would not otherwise receive statins".

Gregory B. Lim

ORIGINAL ARTICLE Vieira-Silva, S. et al. Statin therapy is associated with lower prevalence of gut microbiota dysbiosis. Nature https://doi.org/ 10.1038/s41586-020-2269-x (2020) RELATED ARTICLE Tang, W. H. W. et al. Dietary metabolism, the gut microbiome, and heart failure. Nat. Rev. Cardiol. 16, 137-154 (2019) 